\title{
Specific and global regulation of mRNA stability during osmotic stress in Saccharomyces cerevisiae
}

\author{
LORENA ROMERO-SANTACREU, JOAQUÍN MORENO, JOSÉ E. PÉREZ-ORTíN, and PAULA ALEPUZ \\ Departamento de Bioquímica y Biología Molecular, Facultad de Ciencias Biológicas, Universitat de València, E-46100 Burjassot, Spain
}

\begin{abstract}
Hyperosmotic stress yields reprogramming of gene expression in Saccharomyces cerevisiae cells. Most of this response is orchestrated by Hog1, a stress-activated, mitogen-activated protein kinase (MAPK) homologous to human p38. We investigated, on a genomic scale, the contribution of changes in transcription rates and mRNA stabilities to the modulation of mRNA amounts during the response to osmotic stress in wild-type and hog1 mutant cells. Mild osmotic shock induces a broad mRNA destabilization; however, osmo-mRNAs are up-regulated by increasing both transcription rates and mRNA half-lives. In contrast, mild or severe osmotic stress in hog1 mutants, or severe osmotic stress in wild-type cells, yields global mRNA stabilization and sequestration of mRNAs into P-bodies. After adaptation, the absence of Hog1 affects the kinetics of P-bodies disassembly and the return of mRNAs to translation. Our results indicate that regulation of mRNA turnover contributes to coordinate gene expression upon osmotic stress, and that there are both specific and global controls of mRNA stability depending on the strength of the osmotic stress.
\end{abstract}

Keywords: osmotic stress; Hog1 MAPK; transcription rates; mRNA stability; P-bodies

\section{INTRODUCTION}

Cellular responses to external signals usually include important changes in gene expression patterns. Reprogramming the amount of proteins will depend, among others, on the variations of the abundance of the corresponding mRNAs (RA), which can be changed by modifying gene transcription rates (TR) and/or transcript stabilities (PérezOrtín et al. 2007). To date, transcription has been the main topic of gene expression studies. However, to understand how gene expression is globally modified by external signals with the adequate intensity and timing, it is necessary to study the relative contribution of transcriptional and posttranscriptional mechanisms (Mata et al. 2005; Garneau et al. 2007).

One feasible model for the global study of gene expression is the response of the yeast Saccharomyces cerevisiae to osmotic stress. Hyperosmotic stress causes an immediate dissociation of most proteins from chromatin and a transient inhibition of translation initiation in yeast cells

Reprint requests to: Paula Alepuz, Departamento de Bioquímica y Biología Molecular, Facultad de Ciencias Biológicas, Universitat de València, C/Dr. Moliner 50, E-46100 Burjassot, Spain; e-mail: paula. alepuz@uv.es; fax: 34-96-3544635.

Article published online ahead of print. Article and publication date are at http://www.rnajournal.org/cgi/doi/10.1261/rna.1435709.
(Uesono and Toh-E 2002; Proft and Struhl 2004). The HOG MAPK pathway is also rapidly activated by osmotic stress through three different membrane sensor systems (de Nadal et al. 2002; O'Rourke and Herskowitz 2002). p38-Homologous MAPK Hog1 is responsible for most of the adaptative responses to osmotic stress in S. cerevisae cells. First, Hog1 modulates the activity of several ion membrane transporters (Proft and Struhl 2004). Second, Hogl mediates cell-cycle arrest in G1 phase (Escote et al. 2004). Third, Hog1 regulates the reprogramming of global gene expression (Posas et al. 2000; Rep et al. 2000). And fourth, Hogl is required for the re-initiation of translation after the initial stop (Uesono and Toh-E 2002).

Different genomic studies have analyzed the changes in mRNA levels after an osmotic shock (Gasch et al. 2000; Posas et al. 2000; Rep et al. 2000). The RA of around 7\% of yeast genes increased significantly after mild osmotic stress treatments, showing a rapid and transient induction pattern that was, in most cases, Hog1 dependent. Stronger osmotic stress conditions resulted in the increase of a similar set of mRNAs, although the response was delayed. Besides, a reduction in the levels of an important number of yeast mRNAs was noted after an osmotic shock (Melamed et al. 2008). Most of this reduction is Hog1 dependent, e.g., the drop in the RA of many ribosomal proteins (Uesono and Toh-E 2002). Several transcription 
factors (Hot1, Sko1, Msn2, Msn4, and Smp1) seem to act downstream to Hogl and control different subsets of osmotic-induced mRNAs (Hohmann et al. 2007). Moreover, Hog1 itself associates with chromatin at the promoters of osmotic-sensitive genes to induce transcription (Alepuz et al. 2001, 2003; Pascual-Ahuir et al. 2006; Pokholok et al. 2006). Additionally, Hog1 behaves as a transcriptional elongation factor for genes induced upon osmotic stress (Pokholok et al. 2006; Proft et al. 2006).

In response to stress, global and mRNA-specific studies have revealed the importance of mRNA turnover in governing gene expression. In human cells, Fan et al. (2002) demonstrated that around $50 \%$ of stress genes were regulated at the level of mRNA stability. In yeast, a genomewide analysis of mRNA stability suggested that mRNA decay could be a major mediator in the response to heatshock and nutrient deprivation (Grigull et al. 2004). The genomic studies of García-Martínez et al. (2004) and Molina-Navarro et al. (2008) analyzed changes in yeast TR and mRNA half-lives during a shift from glucose to galactose and in response to oxidative stress. They concluded that for some functional groups of genes, changes in mRNA stability play an important role in the adaptation to new environmental conditions. In response to severe osmotic shock, examining the decay of specific yeast mRNAs revealed a stabilization of the transcripts (Greatrix and van Vuuren 2006; Hilgers et al. 2006). This mRNA stabilization could be due to the inhibition of deadenylation (Hilgers et al. 2006), which was also observed in mammalian cells after osmotic shock (Gowrishankar et al. 2006). In $S$. cerevisiae, deadenylation of the $3^{\prime}$-poly (A) tail is the first step of mRNA degradation, after which the decapping and mRNA hydrolysis by the $5^{\prime}-3^{\prime}$ exonuclease Xrn1 or a $3^{\prime}-5^{\prime}$ degradation by the exonucleolytic activity of the exosome follows. Regulation of mRNA decay is driven through mRNA binding proteins (RBP), which interact with the decay machinery (Parker and Sheth 2007) and may control groups of functionally related genes denominated "posttranscriptional operons" or "decay regulons" (Keene and Tenenbaum 2002; Wang et al. 2002).

In the last few years, the modulation of the stability of target mRNAs has been discovered to be one of the mechanisms used by MAP kinases to control gene expression in response to cellular stimuli. The mammalian Hog1 homologous, p38, stabilizes the cytokine mRNAs regulating the binding of destabilizing factor TTP (tristretraprolin) to the AU-rich elements (ARE) in the $3^{\prime}$-untranslated regions (3'UTR) (Sandler and Stoecklin 2008). Notably, the yeast mRNA TIF51A, whose stability is regulated by a carbon source through its ARE, is destabilized when Hog1 is inhibited (Vasudevan and Peltz 2001). The Hog1-homologous in fission yeast, Sty1, plays a role in the control of the mRNA stability of Atf1, a transcription factor that coordinates the expression of many stress genes in response to oxidative stress (Rodríguez-Gabriel et al. 2003).
As already mentioned, one of the early responses upon osmotic shock is the inhibition of translation (Uesono and Toh-E 2002). For several stresses, a decrease in translation rates correlates with an increase in P-bodies, where mRNA decay factors concentrate and mRNA decay can occur (Teixeira et al. 2005; Parker and Sheth 2007). Indeed, after a short time of severe osmotic stress, an increase in P-bodies was observed in wild-type yeast cells, and this increase significantly reduced in a strain lacking Hog1. This increase has been suggested to be the result of the incorporation of mRNAs from the polysome pool, which could prove beneficial for the cell (Teixeira et al. 2005).

In this work we have investigated, at genomic scale, the contribution of changes in TR and mRNA stabilities to the reprogramming of gene expression after osmotic shock in $S$. cerevisiae cells. The results show that stability is extensively modified for mRNAs in response to osmotic stress. For most genes, TR and mRNA turnover changed unidirectionally to increase or decrease RA. Mild osmotic shock induced the specific stabilization of the mRNAs involved in the response to osmotic stress (osmo-mRNAs), while most mRNAs were destabilized. Conversely, a severe osmotic shock yielded a global and Hog1-independent stabilization of mRNAs, which moved to P-bodies. After adaptation to stress, P-bodies decreased in wild-type cells, but remained longer in the hog1 mutants. Our results suggest that two different responses regulate the cytoplasmic fate of the mRNAs in accordance with the strength of the osmotic stress.

\section{RESULTS}

\section{Global kinetic analysis of transcription rates, mRNA levels, and mRNA stabilities after osmotic stress}

The global transcriptional response to osmotic stress has been thoroughly studied in the yeast S. cerevisiae. However, the respective contributions of TR and mRNA stabilities to the RA change are yet to be determined. Since TR can be calculated on a genomic scale in S. cerevisiae cells (GarcíaMartínez et al. 2004), our first objective was to determine how TR of all yeast genes are modified in response to osmotic stress and how TR changes correlate with RA changes for each specific gene. Additionally, we examined the contribution of the osmo-stress Hog1 MAPK to these changes. For this purpose, we determined the TR and RA for all yeast genes at $0,2,4,6,8,10$, and $15 \mathrm{~min}$ after osmotic stress yielded by $0.4 \mathrm{M} \mathrm{NaCl}$ using the genomic run-on (GRO) method (García-Martínez et al. 2004). After 2 min of stress, global TR in wild-type (wt) cells was reduced by $50 \%$, and to a lesser extent in hog 1 mutant cells (Fig. 1A). Then, a recovery of the TR in wt cells followed, which increased by more than $50 \%$ in relation to the initial value after $8 \mathrm{~min}$ of stress. However, global TR recovered 
A

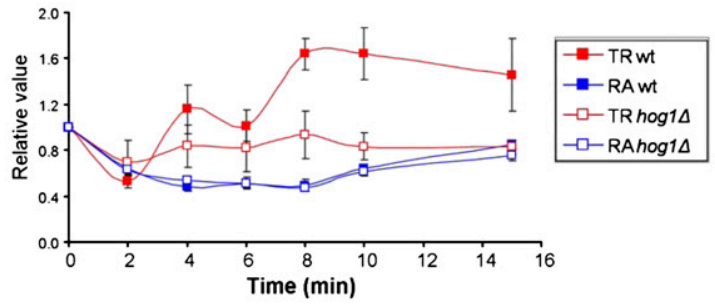

B

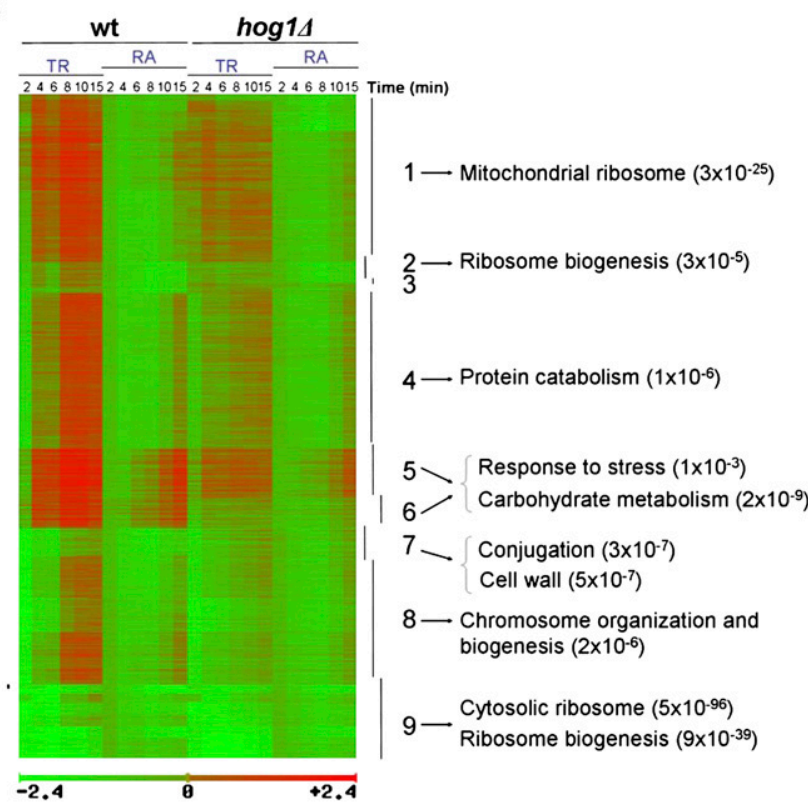

C

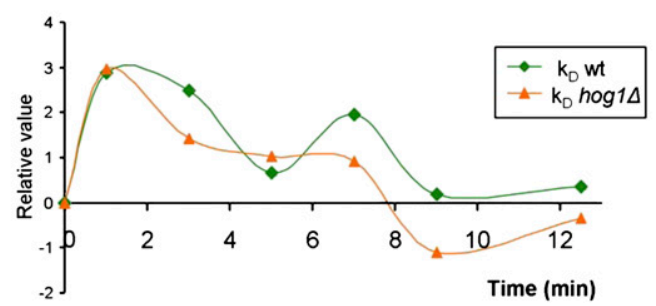

FIGURE 1. Changes in RNA polymerase II TR and RA during the response to osmotic stress. Wild-type (wt) and hog $1 \Delta$ cells were grown in YPD until exponential phase and then treated with $0.4 \mathrm{M} \mathrm{NaCl}$. Samples taken at $0,2,4,6,8,10$, and 15 min of osmotic shock were processed to measure TR and RA of all yeast genes. $(A)$ Changes in global TR and RA after osmotic shock. These two parameters were normalized to an arbitrary value of 1 at $t=0$. Error bars represent the standard error of three independent experiments. (B) Clustering of genes according to their TR and RA profiles. Data set series are referred to their respective time 0 in logarithm scale. Relative repression is shown in green and relative induction in red. The most significant gene functional categories for each cluster are shown with their $P$-values. $(C)$ Changes in global $k_{\mathrm{D}}$ values in response to osmotic stress. The graph represents the difference between the mean $k_{\mathrm{D}}$ value for all genes at each indicated time and the mean $k_{\mathrm{D}}$ value at time 0 , divided by mean $k_{\mathrm{D}}$ value at time 0 . Standard error of the normalized $k_{\mathrm{D}}$ deviations was between 0.02 and 0.05 for all time points. more slowly in hog1 cells and did not reach the initial level after 15 min of stress. The defect of hogl in recovering TR rapidly after osmotic shock may be due to the need of a functional Hog1 to rapidly modify the membrane Nha1 $\mathrm{Na}^{+} / \mathrm{H}^{+}$antiporter and the Tok1 potassium channel to allow a rapid reassociation of proteins with chromatin (Proft and Struhl 2004). Regarding the global mRNA amounts, in the wt strain a decrease is observed with a minimum, $50 \%$ of the initial value, after $8 \mathrm{~min}$ of stress, and then it slowly recovered. Surprisingly, and despite the differences in TR kinetics between wild-type and hog1 cells, the global RA profiles were very similar for both strains. This result suggests that changes in mRNA stability could play an important role in at least one of the strains in response to osmotic stress.

In order to identify whether genes with a related function present similar variations in TR and RA, we performed a clustering of all yeast genes according to their TR and RA profiles (Fig. 1B). A general feature is that the changes in TR are more intense and faster than those in RA. We classified genes in nine different clusters. Most were statistically enriched in functional categories (a comprehensive genomic study will be described elsewhere). Mitochondrial genes were over-represented in Cluster 1, and their TR and RA profiles were similar to the global ones. The group of ribosomal proteins was mainly over-represented in Cluster 9. The genes of this cluster showed a continuous decrease of TR and RA, which was still observed after 15 min (Fig. 1B). Our gene clustering allows the identification of two groups of genes, Clusters 5 and 6, which are transcriptionally induced by osmotic stress (increases in TR and RA). "Stress response" genes and genes of the "carbohydrate metabolism" are over-represented in these two clusters. The transcriptional induction was partially dependent on Hog1 for genes of Cluster 5 and was completely dependent on the MAPK for genes of Cluster 6.

Our results show, for the first time, that transcription rates are importantly and globally modified by osmotic stress. As expected, TR changes take place before a modification is observed in the RA (Pérez-Ortín et al. 2007). In general, TR and RA variations correlated, but a few exceptions were observed. One such exception was noted for genes of Cluster 7, which was enriched in genes of "cell wall formation." For these genes, while TR increased after the initial drop, RA slightly decreased, thus suggesting that changes in mRNA stability could play a role in the regulation of these genes.

Next, we decided to determine the contribution of changes in mRNA stability to the changes in RA. Previously (Pérez-Ortín et al. 2007), we developed a mathematic algorithm to infer mRNA stability from the TR and RA data for situations of rapid and transient changes, like those occurring during osmotic shock produced by $0.4 \mathrm{M} \mathrm{NaCl}$. We used this algorithm to calculate the global changes in mRNA stability expressed as variations in the mRNA 
degradation constant $\left(k_{\mathrm{D}}\right)$ (Fig. 1C). For the first 1-2 min of osmotic shock, a global mRNA destabilization was noted in both wt and hog1 mutant cells. Global mRNA stability returns to initial levels after about 9 min in wt cells. After this time, however, an increase of global transcript stability in hog1 mutants was observed. The immediate destabilization of mRNAs estimated for wt and hogl cells leads to the rapid drop in global RA amounts detected for both strains after 2 min of stress (Fig. 1A). Afterward, the differential mRNA stabilization observed in hog1 at longer times compensates for a higher TR profile in the wt strain to produce a similar evolution of global RA in both strains (Fig. 1A). Therefore, our calculations of mRNA stabilities, even at the global level, show that osmotic stress causes important changes in mRNA decay, which are determinant to explain the RA variations observed. In addition, they show that after the first minutes of osmotic stress, mRNA stability is regulated differently for wt and hog1 cells.

\section{Osmo-mRNAs are specifically stabilized in response to mild osmotic stress}

Our genomic transcriptional data showed that, in response to osmotic shock, changes in RA were mostly preceded by changes in TR (Fig. 1B). We wondered if the variation in the TR of each specific gene was sufficient to explain the variation observed in its mRNA level without changing mRNA stability. To that end, we compared the experimental RA changes observed during the osmotic shock time course with the theoretical RA changes calculated from the experimental TR, assuming that the initial mRNA stability $\left(k_{\mathrm{D}}\right)$ did not change under stress (see Materials and Methods). Using the difference between the experimental and theoretical RA, yeast genes were classified according to the influence that their mRNA stabilities have on RA changes (Fig. 2A). Out of 5322 genes analyzed, 12\% of them (656 genes) did not display important changes in mRNA stability during osmotic shock with $0.4 \mathrm{M} \mathrm{NaCl}$ since their stability indexes were close to 0 (values between -1 and +1 ). However, the histogram displayed an asymmetric distribution of genes where $85 \%$ of the mRNAs were destabilized to some extent during the $15 \mathrm{~min}$ of stress (4545 genes present a stability index $<-1$ ). This result indicates a global destabilization trend of yeast mRNAs in response to a mild osmotic stress and explains why the global RA profile of wild-type cells does not increase during osmotic stress even though the global TR profile does (Fig. 1A). Interestingly, the mRNAs of 121 genes (2.2\%) showed high stabilization indexes (>1) (Supplemental Table 1). We searched for gene functional groups (FuncAssociate) within the 121 genes whose mRNAs were stabilized and discovered that the stress response $\left(P\right.$-value $\left.5.9 \times 10^{-6}\right)$ and "alphatrehalose-phosphate synthase complex" ( $P$-value $4.6 \times$ $10^{-5}$ ) categories were over-represented. Remarkably, stress response proteins and proteins involved in the production
A

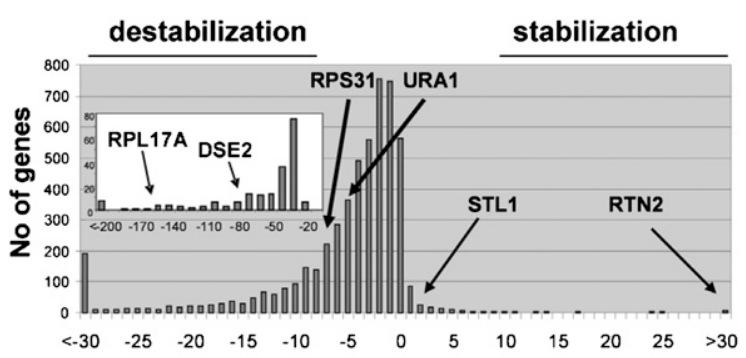

B

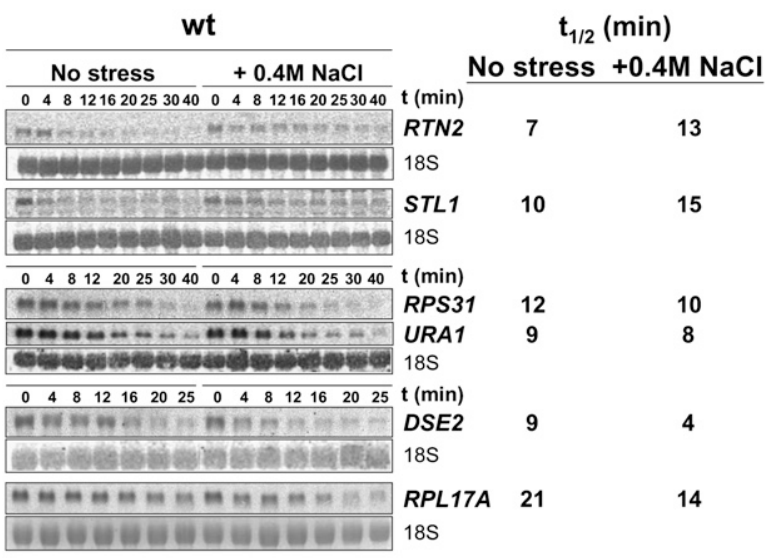

C

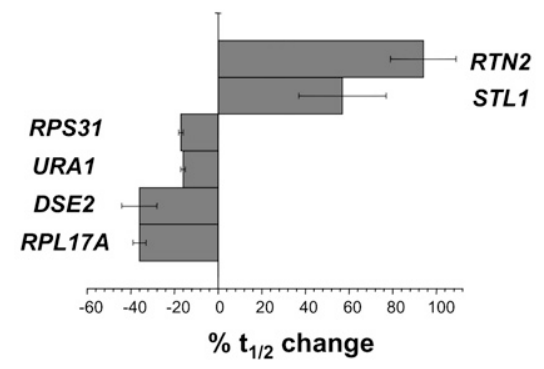

FIGURE 2. Specific stabilization of osmo-mRNAs during mild osmotic stress. (A) Global distribution of genes according to their destabilization $(<0)$ or stabilization $(>0)$ indexes (see Materials and Methods). Arrows in the histogram indicate the position of the genes whose mRNA half-lives have been checked by conventional methods. (B) Determination of mRNAs half-lives $\left(t_{1 / 2}\right)$ before and after mild osmotic stress. Strains PAY500 $\left(r p b 1-1^{t s}\right)$, PAY498 $\left(r p b 1-1^{t s}\right.$ tet $\mathrm{O}_{2}-\mathrm{RTN2}$ ), and PAY495 ( $r p b 1-1^{\text {ts }}$ tet $\left.\mathrm{O}_{2}-\mathrm{STL} 1\right)$ were grown in YPD until exponential phase and, before or after treatment for 5 min with $\mathrm{NaCl} 0.4 \mathrm{M}$, transcription was stopped by shifting the cells to $37^{\circ} \mathrm{C}$ and adding doxycycline $(10 \mu \mathrm{g} / \mathrm{mL})$. Aliquots were taken at the indicated times and RA of the indicated genes were determined by Northern analysis (using rRNA18S signal as a loading control). The half-life value of a representative experiment is shown. (C) mRNA half-life change after mild osmotic stress. Mean half-life change (\%) for the indicated mRNAs was calculated from at least three independent experiments. Error bars represent the standard deviation.

of trehalose are necessary to survive osmotic stress, and these two functional categories were also found to be upregulated in global expression experiments (i.e., RA data) under osmotic stress (our results, and those of Posas et al. 
[2000], Rep et al. [2000], and Hohmann et al. [2007]). Conversely, genes of the "cytosolic ribosome" ( $P$-value $\left.9 \times 10^{-48}\right)$ and "cell wall components" $(P$-value $5 \times$ $\left.10^{-11}\right)$ categories were over-represented among the 200 genes whose mRNAs were most destabilized $(<-29$; Supplemental Table 2).

Stabilization of mRNAs could be positively correlated or not with an increase in the RA and/or TR (García-Martínez et al. 2004). We found that 75\% (91 genes) of the genes whose mRNAs were stabilized also showed a significant increase in their RA after osmotic stress. Of these induced genes, 93\% (85 genes) also presented an increase in their TR. Therefore, mRNA stabilization cooperates with transcription induction to increase mRNA levels of some osmostress genes. Interestingly, this group of genes was enriched in targets of the stress-response transcription factors Msn2/ Msn4 ( $P$-value $10^{-10}$; Hohmann et al. 2007). This result could indicate an effect of these two factors at both the transcriptional and mRNA stability levels.

To verify our global predictions of changes in mRNA stability, we checked mRNA half-lives of several representative genes by conventional methods. We chose STL1 and RTN2 osmo-mRNAs, predicted to be stabilized by our indirect analysis, and the putatively destabilized mRNAs RPL17A and RPS31 (encoding for ribosomal proteins), DSE2 (involved in cell wall formation), and URA1 (involved in the metabolism of nucleotides) (Fig. 2A). Since STL1 and RTN2 mRNAs are barely detectable in nonstress conditions, we expressed these two genes under the tet $\mathrm{O}_{2}$ promoter, which allowed us to measure their mRNA half-lives in nonstress conditions and, additionally, to shut off transcription by adding doxycycline (Bellí et al. 1998). Furthermore, all yeast strains used in the experiments to measure mRNA half-lives contained the thermosensitive mutation $r p b 1-1$. Therefore, general transcription was shut off by incubating the cells at $37^{\circ} \mathrm{C}$ plus adding doxycycline and this allowed measuring mRNA half-lives of other genes under their own promoter. For stress-regulated genes, the switching of the native promoters to tetO $\mathrm{O}_{2}$ promoter not only allowed for RA determinations in nonstress conditions, but also avoided the transcriptional up-regulation that could result from incubating cells at $37^{\circ} \mathrm{C}$.

As shown in Figure 2B, the osmo-mRNAs RTN2 and STL1 were stabilized when yeast cells were treated with 0.4 $\mathrm{M} \mathrm{NaCl}$. The RTN2 half-life increase was higher than the STL1 half-life increase (Fig. 2C; 94\% and 53\%, respectively) in accordance with our prediction. Conversely, the mRNA half-lives for RPL17A, RPS31, DSE2, and URA1 decreased during the osmotic shock, matching again our predictions (Fig. 2B,C). Other mRNAs inducible by osmotic stress or belonging to other functional categories were also tested and showed half-life variations in accordance with our predictions (Supplemental Fig. 1). Thus, these results confirm a specific stabilization of osmo-mRNAs.

\section{Hog1 deletion provokes stabilization of many mRNAs in response to mild osmotic stress}

STL1 and RTN2 genes show increases in TR and mRNA stability in response to osmotic stress. For both genes, TR induction is completely controlled by Hogl (data not shown). We wondered if the stress activated Hog1 also regulates the specific mRNA stabilization of osmo-mRNAs. To answer this, we measured the changes in RTN2 mRNA half-life in response to treatment with $0.4 \mathrm{M} \mathrm{NaCl}$ in a hog1 deletion strain. We found that RTN2 half-life is also increased in the hogl mutant strain under osmotic stress (Fig. 3). Next in hog1 mutant, we checked the variations in mRNA half-lives of RPS31, URA1, and DSE2 mRNAs, which are destabilized in a wt strain under $0.4 \mathrm{M} \mathrm{NaCl}$ (Fig. 2). All three mRNAs were stabilized in hogl cells and their half-lives underwent important increases (Fig. 3). These results suggest that a general and nonspecific mRNA stabilization occurs in response to osmotic stress when Hogl does not act in yeast cells, as predicted by the global stabilization of transcripts in hogl cells after the first minutes of stress (Fig. 1C).

Our results indicate that mild osmotic stress yields two different outputs in wt and hog1 strains. In the former, most mRNAs are destabilized and osmo-mRNAs are specifically stabilized. In the latter, a global mRNA stabilization takes place. The general increase in mRNA halflives in hogl precludes the determination of whether the

A

\begin{tabular}{|c|c|c|c|}
\hline hog14 & & \multicolumn{2}{|c|}{$t_{1 / 2}(\min )$} \\
\hline No stress $\quad+0.4 \mathrm{M} \mathrm{NaCl}$ & & No stress & $+0.4 \mathrm{M} \mathrm{NaCl}$ \\
\hline 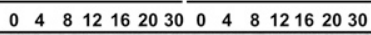 & $t(\min )$ & & \\
\hline 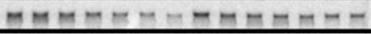 & RTN2 & 10 & 15 \\
\hline 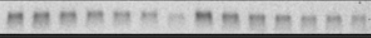 & RPS31 & 12 & 24 \\
\hline 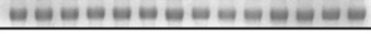 & $18 \mathrm{~S}$ & & \\
\hline 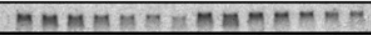 & URA1 & 12 & 22 \\
\hline 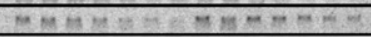 & DSE2 & 12 & 19 \\
\hline 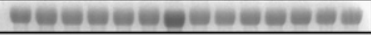 & $25 S$ & & \\
\hline
\end{tabular}

B

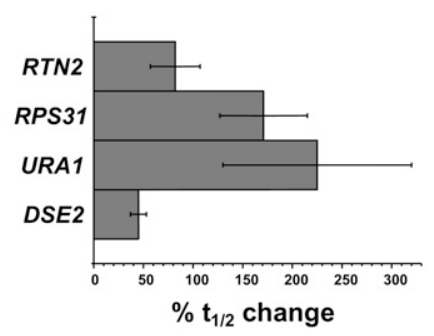

FIGURE 3. General mRNA stabilization in hog1s mutant after treatment with $0.4 \mathrm{M} \mathrm{NaCl}$. (A) Half-lives of RPS31, URA1, and DSE2 mRNAs in strain PAY532 (rpbl-1 $1^{\text {ts }}$ hog $\left.1 \Delta\right)$ and of RTN2 mRNA in strain PAY591 ( $r p b 1-1^{t s}$ hog1s rtn2s) containing p416TEF-RTN2 were measured as described in Figure 2. The half-life value of a representative experiment is shown. (B) mRNA half-life change (\%) after mild osmotic stress in hog1d strain. Means were calculated from at least three independent experiments. Error bars represent the standard deviation. 
osmo-mRNA specific stabilization under mild osmotic stress depends on Hog1.

\section{Severe osmotic stress produces a global mRNA stabilization that is Hog1 independent}

Other authors have reported the stabilization of some mRNAs in osmotic-stressed yeast cells (Greatrix et al. 2006; Hilgers et al. 2006). In their experiments, transcripts that are not regulated transcriptionally by stress were stabilized by severe osmotic stress with either $1 \mathrm{M} \mathrm{KCl}$ or $40 \%$ glucose. Here, we have reported the specific mRNA stabilization of osmotic stress-regulated genes and the destabilization for most yeast mRNAs under mild osmotic stress conditions with $0.4 \mathrm{M} \mathrm{NaCl}$. These different observations would not be contradictory if mRNA stability were regulated differently according to the strength of the osmotic shock. To test this possibility, we measured halflife variations of the mild-osmotic stress stabilized RTN2 and destabilized RPS31, URA1, and DSE2 mRNAs under severe osmotic stress caused by $1 \mathrm{M} \mathrm{KCl}$. We observed that the four mRNAs were strongly stabilized with half-life increases of more than fivefold (Fig. 4). These results are consistent with a general and nonspecific stabilization under severe osmotic stress, as already suggested (Greatrix et al. 2006; Hilgers et al. 2006). Moreover, these results indicate that stability of some mRNAs (RPS31, URA1, and

A

\begin{tabular}{|c|c|c|c|}
\hline wt & & \multicolumn{2}{|c|}{$t_{1 / 2}(\min )$} \\
\hline No stress $\quad+1 \mathrm{M} \mathrm{KCl}$ & & No stress & $+1 \mathrm{M} \mathrm{KCl}$ \\
\hline$0 4 8 8 1 5 2 0 3 0 4 0 \longdiv { 0 4 8 8 1 5 2 0 3 0 4 0 }$ & $t(\min )$ & & \\
\hline 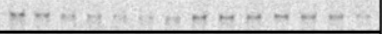 & RTN2 & 10 & 76 \\
\hline 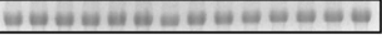 & $18 \mathrm{~S}$ & & \\
\hline ตัต & RPS31 & 14 & 71 \\
\hline 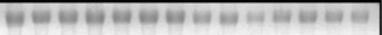 & $18 \mathrm{~S}$ & & \\
\hline 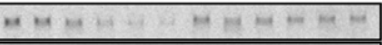 & URA1 & 7 & 77 \\
\hline 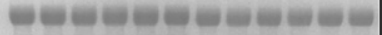 & $25 \mathrm{~S}$ & & \\
\hline 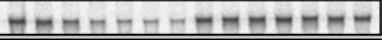 & DSE2 & 8 & $>80$ \\
\hline 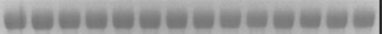 & $18 S$ & & \\
\hline
\end{tabular}

B

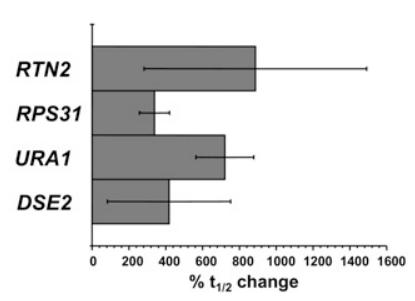

FIGURE 4. General mRNA stabilization during severe osmotic stress (1 M KCl). (A) Half-lives of RPS31, URA1, and DSE2 mRNAs in strain PAY500 (rpb1-1 ${ }^{t s}$ ) and of RTN2 mRNA in strain PAY498 ( $r p b 1$ $1^{\text {ts }}$ tet $\mathrm{O}_{2}$-RTN2) were measured as described in Figure 2 . The half-life value of a representative experiment is shown. (B) Half-life change (\%) after severe osmotic stress in a wt strain. Means were calculated from at least three independent experiments. Error bars represent the standard deviation.
DSE2) is regulated in opposite directions depending on the strength of the osmotic stress condition.

The general mRNA stabilization we observed in hog1 mutant cells resembles the global mRNA stabilization reported for wt cells under severe osmotic stress. One likely explanation of this similar behavior is that hog1 cells under 0.4 $\mathrm{M} \mathrm{NaCl}$ sense stronger stress, since the initial salt extrusion by ion membrane transporters does not occur (Proft and Struhl 2004). We hypothesize that under severe stress conditions, caused by treatment of wt cells with $1 \mathrm{M} \mathrm{KCl}$ or treatment of hog1 cells with $0.4 \mathrm{M} \mathrm{NaCl}$, the same mechanism will induce mRNA stabilization and that this mechanism will be Hog1 independent. Supporting this hypothesis we found that treatment with $1 \mathrm{M} \mathrm{KCl}$ of cells lacking Hogl also induced the mRNA stabilization of all mRNAs checked (Fig. 5). Moreover, our data suggest that the increase in mRNA half-lives correlates positively with the strength of the osmotic stress (Figs. 3-5, cf. increases in mRNA half-lives).

\section{P-bodies increase in wt and hog1 cells correlate with general mRNA stabilization}

In yeast, an increase in the extracellular osmolarity leads to a transient inhibition of translation at the initiation step. After adaptation, protein synthesis is restored through an unknown Hog1-dependent mechanism (Uesono and TohE 2002). Additionally, after $15 \mathrm{~min}$ of exposure to $1 \mathrm{M} \mathrm{KCl}$ there is an increase in P-bodies that is significantly reduced in hog1 mutant cells, and which could be due to the incorporation of mRNAs from polysomes (Teixeira et al. 2005). Based on our results, which show a specific and a general mRNA stabilization in response to different osmotic stress conditions, we presume that osmo-mRNAs stabilized by mild osmotic stress will be engaged into polysomes for translation; meanwhile global mRNAs stabilized by severe osmotic stress will be abducted into a nontranslating pool in P-bodies. To gain insights into the cytoplasmic fate of stabilized mRNA, we examined the status of P-bodies in wild-type and hogl cells under mild and severe osmotic stress. After exposure of wt cells to 0.4 $\mathrm{M} \mathrm{NaCl}$, we observed a transient appearance of faint P-bodies during the interval from 5 to $10 \mathrm{~min}$ of stress (Fig. 6A,C). However, P-bodies assembled in wt cells under severe osmotic stress $(1 \mathrm{M} \mathrm{KCl})$ were more intense and were visualized over a longer interval, from 15 to $90 \mathrm{~min}$ after stress (Fig. 6B,C). These results correlated well with the observation by Uesono and Toh-E (2002) that the translation stop lasts longer when osmotic stress is more severe. The sustained presence of P-bodies under $1 \mathrm{M} \mathrm{KCl}$ also correlated with the global and important increase of mRNA half-lives under such a condition. Altogether, our results and the previous results suggest that translation inhibition, global mRNA stabilization, and P-body formation are closely related. 


\begin{tabular}{|c|c|c|c|}
\hline hog1 $1 \Delta$ & & \multicolumn{2}{|c|}{$t_{1 / 2}(\min )$} \\
\hline No stress $\quad+1 \mathrm{M} \mathrm{KCl}$ & & No stress & $+1 \mathrm{M} \mathrm{KC}$ \\
\hline $\begin{array}{llllllllllll}0 & 4 & 8 & 15 & 20 & 30 & 0 & 10 & 20 & 30 & 40 & 60\end{array}$ & & & \\
\hline 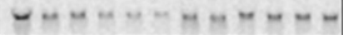 & RTN2 & 12 & $>\mathbf{2 0 0}$ \\
\hline 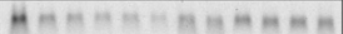 & RPS31 & 13 & $>200$ \\
\hline 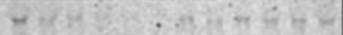 & URA1 & 13 & $>\mathbf{2 0 0}$ \\
\hline " & $25 \mathrm{~S}$ & & \\
\hline UG & DSE2 & 11 & $>\mathbf{2 0 0}$ \\
\hline 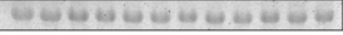 & & & \\
\hline
\end{tabular}

FIGURE 5. General mRNA stabilization in hog1d mutant during severe osmotic stress (1 M KCl). Half-lives of RTN2, RPS31, URA1, and DSE2

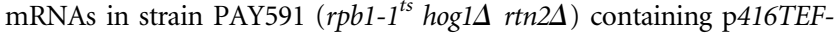
RTN2 were measured as described in Figure 2. The half-life value of a representative experiment, out of three independent experiments, is shown.

Next we tested the formation of P-bodies in hog1 mutant cells. Under mild osmotic stress conditions caused by $0.4 \mathrm{M}$ $\mathrm{NaCl}, \mathrm{P}$-bodies started to be visualized at $5 \mathrm{~min}$ and remained until $30 \mathrm{~min}$ of stress (Fig. 6A,C). This result indicates that $\mathrm{P}$-bodies are assembled in hog1 mutants and, importantly, that P-bodies remain longer than in wt cells. Severe osmotic stress also induced the formation of P-bodies in hog1. Very bright P-bodies were visualized after 45 min of stress, and were still observed with a similar brightness after $4 \mathrm{~h}$ of treatment with $1 \mathrm{M} \mathrm{KCl}$ (Fig. 6B,C; data not shown). These observations indicate that Hog1 is not essential for the stress-induced P-body assembly, but its absence significantly modifies the disassembly kinetics of P-bodies after adaptation to osmotic stress. Our results showed a delay in the assembly of P-bodies in hog1 in relation to wt cells (Fig. 6C); this delay could explain the difference in P-body status between hog1 and wt cells at short times (15 min) of severe osmotic stress described by Teixeira et al. (2005). The fact that P-bodies are assembled and sustained in a hog1 mutant under mild and severe osmotic stress correlate with sustained global RNA stabilization in this mutant (Fig. 1C) and supports the hypothesis of a Hog1-independent mechanism of global mRNA stabilization and abduction of stabilized mRNAs into P-bodies when yeast cells sense severe osmotic stress. The much longer period for the disappearance of P-bodies (Fig. 6C) in hog1 mutant cells, compared to wt cells, could just be the result of a stronger intracellular osmotic stress in the absence of the MAP kinase. However, the fact that there is not only a delay in the kinetics of P-bodies but also a difference in the specific kinetics of P-bodies disappearance (Fig. 6C) suggests a specific role of Hog1 in the disassembly of P-bodies and return of mRNAs to translation.

\section{DISCUSSION}

\section{Global transcriptional and post-transcriptional responses to mild osmotic stress}

In this work we have analyzed global changes in transcription rates, mRNA amounts, and mRNA stabilities in response to mild osmotic stress $(0.4 \mathrm{M} \mathrm{NaCl})$. The determination of these three parameters in the same experiment by means of the GRO method evaluates the relative contributions of transcriptional and post-transcriptional regulation to the global changes in gene expression. Our results indicate that variations in RA are mostly preceded by unidirectional changes in TR, and that mild osmotic stress produces changes in mRNA stabilities of most of the genes ( $82 \%$ of the genes). These changes most frequently involve destabilization (4545 mRNAs), and for a few genes (121 mRNAs), most of them belonging to the functional groups

A

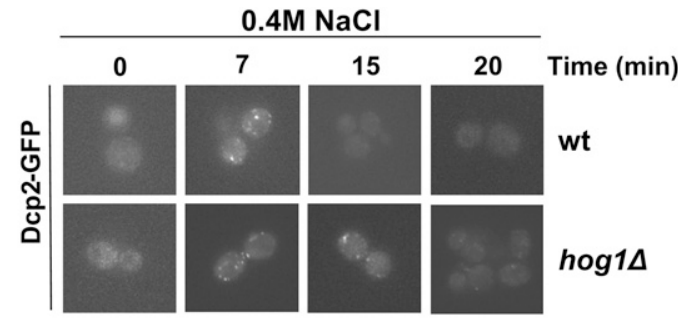

B

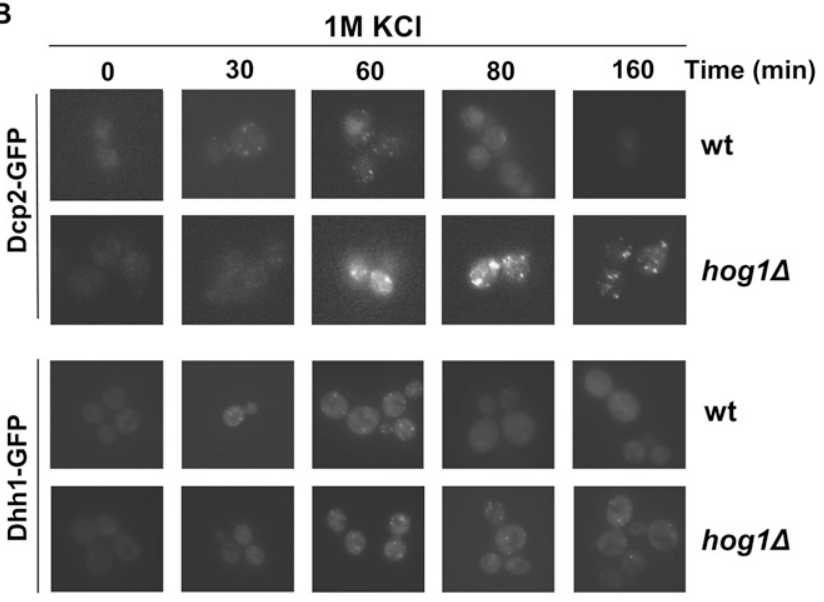

C

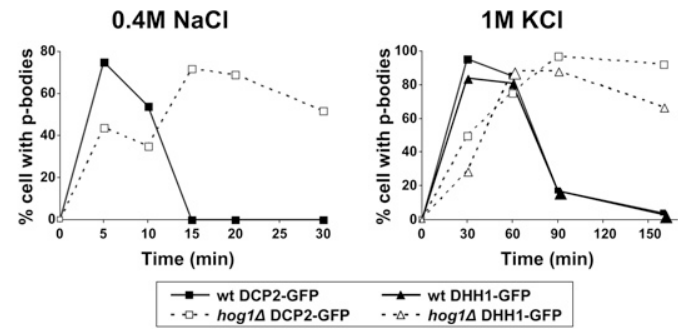

FIGURE 6. Kinetics of P-bodies increase under osmotic shock depends on the stress conditions and the presence of Hog1. Wildtype and hog1 $($ PAY185) strains expressing a GFP-tagged version of the decapping enzyme Dcp2p or BY4741 and BY4741hog1s strains expressing the GFP-tagged version of the decapping activator Dhhlp were grown until exponential phase and then treated with $(A) \mathrm{NaCl}$ $0.4 \mathrm{M}$ or $(B) \mathrm{KCl} 1 \mathrm{M}$. Dcp2-GFP and Dhh1-GFP were used to visualize P-bodies at the indicated times of osmotic stress. (C) Quantification of the percentage of wt or $\operatorname{hog} 1 \Delta$ cells with P-bodies after treatment with $0.4 \mathrm{M} \mathrm{NaCl}$ or $1 \mathrm{M} \mathrm{KCl}$ as indicated above. At least 100 cells were quantified in each condition. 
"stress response" and "trehalose production," mRNA stabilization is observed. Our data indicate that both, increasing TR and stabilizing transcripts, contribute to raising the level of these osmo-mRNAs, and suggest that common factors may exist that regulate both processes, such as the transcription factors Msn2/Msn4 (Hohmann et al. 2007). The kinetic strategy of regulating both TR and mRNA stability of a given specific gene would allow to switch to a new RA level in a rapid and transient mode, as required under stress conditions (Pérez-Ortín et al. 2007). Moreover, for some genes of two functional groups, "stressresponse" and "ribosomal proteins," their regulation by osmotic stress occurs in the same direction at three levels. "Stress genes" increase TR and mRNA stability (this work) and translation efficiency (Melamed et al. 2008), while ribosomal genes decrease TR and destabilize mRNAs (this work) and down-regulate translation (Melamed et al. 2008). These results indicate a potentiation effect at multiple levels of regulation and suggest the existence of a common factor in the coordination of these three processes.

\section{Specific and global regulation of mRNA stability depending on the strength of osmotic shock and the presence of Hog1}

Our results have shown that osmo-mRNAs are stabilized by mild osmotic stress, while most other mRNAs, like those for ribosomal proteins or cell wall components, are destabilized. Two important questions emerge from the specific stabilization of osmo-mRNAs. First, one may wonder if the mRNA stabilization is regulated by the osmotic stress signaling MAPK Hog1. Second, it might be asked whether this specific stabilization occurs under any osmotic stress condition. We could not determine whether the mechanism that renders specific stabilization of osmo-mRNAs is lost in the hog1 mutant or if it still works in a wt strain under severe osmotic stress since a global increase in mRNA half-lives took place in both cases. Different observations argue in favor of a mechanism to control the specific osmo-mRNA stabilization that would involve Hog1. First, most mRNAs stabilized by mild osmotic stress are transcriptionally up-regulated by Hog1 and encode for proteins involved in adaptation to osmotic stress. Second, Hog1 has already been involved in the stabilization of a yeast mRNA (Vasudevan and Peltz 2001). Third, Hog1homologs in other organisms, as p38 in mammals and Sty1 in Schizosaccharomyces pombe, regulate mRNA stability in response to stress (Rodríguez-Gabriel et al. 2003; Sandler and Stoecklin 2008). These data make appealing a model in which, in response to osmotic stress, active Hog1 would induce the stabilization of a specific subset of osmomRNAs. Since this subset is also enriched in Msn2/Msn4 targets, one possibility is that co-regulation of transcription and mRNA stability could be established at the very early stages of transcription in the nucleus when a physical proximity exists between the transcription factors, Hog1 and the newly synthesized mRNA (Alepuz et al. 2001; Pascual-Ahuir et al. 2006; Pokholok et al. 2006).

Stabilization of mRNAs through their $3^{\prime}$ UTR is regulated by RBP that recognize specific cis sequences in this region (Garneau et al. 2007). A search for common motifs in the 3'UTR of all the stabilized osmo-mRNAs or most destabilized mRNAs (e.g., osmo-stress, ribosomal proteins, and cell wall components) neither reveled enrichment in any of the described putative regulatory motifs for mRNA stability (Shalgi et al. 2005), including ARE, nor enrichment in the described targets of RBPs. Conversely and importantly, destabilized mRNAs are enriched in Pub1 targets (28\% of the 200 most destabilized mRNAs by mild osmotic stress, $P$-value $4.7 \times 10^{-11}$ ) (Duttagupta et al. 2005). Since Pub1 was previously shown to regulate the stability of one yeast mRNA in a Hog1-dependent manner (Vasudevan and Peltz 2001), it is a candidate to be an osmotic stress-regulated mRNA destabilization factor.

Changes in mRNA stabilities depend on the strength of osmotic stress. Our results suggest that signaling induced by high intracellular salt concentration, as in wild-type cells treated with $1 \mathrm{M} \mathrm{KCl}$ or hogl cells with $0.4 \mathrm{M} \mathrm{NaCl}$ or $1 \mathrm{M}$ $\mathrm{KCl}$, induces a mechanism to globally stabilize mRNAs that would be, therefore, Hog1 independent. This stabilization of mRNAs may be due to the inhibition of the cytoplasmic deadenylase complexes Ccr4p/Pop2p/Not1p and Pan2p/ Pan3p (Hilgers et al. 2006), as occurs in response to hyperosmolarity and other stresses in mammalian cells (Gowrishankar et al. 2006). Moreover, global stabilization of mRNAs correlates with P-body formation. P-bodies could be visualized for relatively long periods (from 25 min to several hours) only under those conditions involving global mRNA stabilization, like mild or severe osmotic stress in hog1 mutants and severe osmotic stress in wt. These results suggest that nonspecific stabilized mRNAs are retained into P-bodies. As other cellular stresses, osmotic shock inhibits translation. Under mild osmotic stress, translation is inhibited rapidly and for short periods; meanwhile translation inhibition is delayed and remains longer under severe osmotic stress (Uesono and Toh-E 2002). This kinetics of translation inhibition correlates well with the P-body assembly as seen in our experiments (Fig. 6). Moreover, translation is not recovered in hog1 mutants and we have observed that P-bodies remain assembled in the mutant cells for hours. All these results suggest that severe osmotic stress induces a Hog1-independent mechanism that yields translation inhibition, mRNA stabilization, and movement of stabilized mRNAs into P-bodies. The fact that correlations exist among these three processes in response to other severe cellular stresses (Kawai et al. 2004; Teixeira et al. 2005; Bhattacharyya et al. 2006; Hilgers et al. 2006) argues in favor of a regulated mechanism of general stress protection. However, it cannot be ruled out that high intracellular salt concentrations could simply alter 
the properties of mRNA-protein complexes and, thus, impair the activity of translation and degradation complexes over mRNAs.

Finally, the question of the biological significance of mRNA stabilization emerges. Several authors have proposed that packaging of mRNAs into P-bodies under stress would regulate gene expression by delaying the translation of retained transcripts to allow the preferential translation of newly synthesized mRNAs encoding proteins that alleviate the stress condition (Bond 2006; Hilgers et al. 2006; Kedersha and Anderson 2007). After adaptation to stress, mRNAs could exit P-bodies and return to translation (Brengues et al. 2005; Bhattacharyya et al. 2006). In the light of this model, we propose that stabilization of osmomRNAs would have a different function than the stabilization of other mRNAs. In the first case, osmo-mRNAs specifically stabilized would be engaged into translation to produce stress protective proteins, whereas in the second case, the cytoplasmic mRNAs that undergo general stabilization under severe osmotic stress would remain in a nontranslating pool. Two recent observations support this idea. First, under mild osmotic stress $(0.4 \mathrm{M} \mathrm{NaCl})$ polysomal fractions are preferentially enriched in osmostress mRNAs (P. Sunnerhagen, pers. comm.). Second, severe osmotic stress $(1 \mathrm{M} \mathrm{NaCl})$ induces an increase in mRNA amounts of many genes, but most of the mRNAs are found in a nonpolysomal fraction, while stress responsive genes are still translationally up-regulated (Melamed et al. 2008).

In summary, this work contributes to the understanding of how external signals regulate gene expression at many levels, such as the TR and the cytoplasmic fate of mRNAs. Under compromised survival, such as osmotic stress, the expression of genes encoding protective proteins could be potentiated by the coordinated up-regulation of transcription, mRNA stability, and translation rates. The factors involved in the coordination of these processes remain to be discovered.

\section{MATERIALS AND METHODS}

\section{Yeast strains and growth conditions}

All strains used were derivatives of wild-type S. cerevisiae W3031A (MATa ura1-3 ade2-1 leu2-3,112 trp1-1) and are listed in Supplemental Table S3. Cells were grown at $30^{\circ} \mathrm{C}$ in YPD medium or SC medium plus $2 \%$ glucose and the required amino acids. Osmotic stress conditions were created by addition of $\mathrm{NaCl}$ at 0.4 $\mathrm{M}$ or $\mathrm{KCl}$ at $1 \mathrm{M}$. For measurement of mRNA decay, transcription was stopped by shifting the cells to $37^{\circ} \mathrm{C}$ and/or adding doxycycline $(10 \mu \mathrm{g} / \mathrm{mL})$.

\section{Plasmids}

p416TEF-RTN2 was constructed by cloning the RTN2 ORF plus 3'UTR (300 base pairs) generated as a BamHI-SalI PCR fragment into the plasmid p416TEF (URA3, CEN6/ARSH4N; Mumberg et al. 1995). To visualize P-bodies by microscopy, a plasmid including DCP2-GFP (CEN, LEU2) or Dhh1-GFP (CEN, LEU2) was used (Sheth and Parker 2003). Yeast strains were transformed by standard techniques and plasmids were maintained by growth in selective media.

\section{Genomic run-on}

GRO experiments with yeast cells grown until exponential phases were carried out according to García-Martínez et al. (2004). Each experiment was repeated three times. To facilitate further normalizations, we used a dot-blot procedure to estimate the proportion of poly(A) mRNA/cell. The total RNA/cell was invariable throughout the experiment. The proportion of poly(A)/total RNA was taken as the estimation for total RA per cell. Each time point determination was obtained from the averaged results of three independent filters. cDNA and GRO hybridizations were normalized as described in García-Martínez et al. (2004).

\section{Global mRNA stability analysis}

For calculation of the theoretical mRNA profile RAs were assumed to be at a steady state at the onset of stress (V. Pelechano and J.E. Pérez-Ortín, unpubl.). Therefore, the initial (steady-state) $k_{\mathrm{D}}$ was calculated as the ratio of TR to RA values determined at time 0 . After the onset of stress, under (presumably) non-steady-state conditions, the theoretical mRNA profile, assuming $k_{\mathrm{D}}$ invariable throughout the time course of the response to osmotic stress, was calculated from the experimental values of TR. Assuming a linear variation of TR between experimentally determined values, the following relation between TR, RA, and $k_{\mathrm{D}}$ has been demonstrated to hold (Pérez-Ortín et al. 2007):

$$
\begin{aligned}
m_{i+1}= & 1 / k_{\mathrm{D}}^{2}\left\langle k_{D} \mathrm{TR}_{i+1}-\left(\mathrm{TR}_{i+1}-\mathrm{TR}_{i}\right) /\left(t_{i+1}-t_{i}\right)\right. \\
& +\left[\left(\mathrm{TR}_{i+1}-\mathrm{TR}_{i}\right) /\left(t_{i+1}-t_{i}\right)-k_{\mathrm{D}} \mathrm{TR}_{i}+k_{\mathrm{D}}^{2} m_{i}\right] \\
& \left.\exp \left[-k_{D}\left(t_{i+1}-t_{i}\right)\right]\right\rangle,
\end{aligned}
$$

where $\mathrm{TR}_{i}$ and $\mathrm{TR}_{i+1}$, are the experimental values for $\mathrm{TR}$ at successive time points $t_{i}$ and $t_{i+1} ; m_{1}$ is the experimental value for RA at time $t_{1}$ and $m_{i}, m_{i+1}$ are the theoretical values of RA at $t_{i}$, $t_{i+1}$. The experimental mRNA profile area was subtracted from the theoretical mRNA profile area to give an index for each gene. Positive indexes indicated mRNA stabilization and negative indexes indicated mRNA destabilization.

\section{Clustering procedures and databases used}

Changes in TR and RA for all yeast genes were evaluated by cluster analysis of normalized averaged values. For cluster analysis of the results, we used the Gene Expression Pattern Analysis Suite v 3.1 (http://www.gepas.org) included in the web server of CIPF Bioinformatic Unit. To test the potential enrichment in gene ontology (GO) categories in the different groups obtained in this study, we used the FuncAssociate (http://llama.med.harvard.edu/ cgi/func/funcassociate) and the FunSpec servers (http://funspec. med.utoronto.ca). The transcription factor search was carried out using the YEASTRACT database (http://www.yeastract.com) that includes documented association between a transcription factor and the target gene. Enrichment in described targets of RBPs 
was carried out using the database UTResource (http://www.ba. itb.cnr.it/BIG/UTRHome/).

\section{Northern blot analysis}

Total RNA was isolated from YPD or SC grown yeast cells that were treated as indicated. The probes used were PCR fragments containing the entire open reading frames of RTN2, URA1, STL1, RPS31, DSE2, and RPL17A. Signals were quantified using a FujiFilm PhosphorImager scanner (FLA-3000).

\section{Preparation of cells for microscopy and quantification of cells with P-bodies}

Cells were grown overnight in the appropriate medium to keep Dcp2-GFP or Dhh1-GFP plasmid (Sheth and Parker 2003). Then, cells were transferred to YPD and grown to an $\mathrm{OD}_{600}$ of 0.5-0.6. Cells were washed and resuspended in SC plus amino acids, without salt or with $0.4 \mathrm{M} \mathrm{NaCl}$ or $1 \mathrm{M} \mathrm{KCl}$. Observations were made at the indicated times using an Axio Imager $\mathrm{Z} 1$ fluorescence microscope (Zeiss). For each experiment and time point indicated, a quantitative analysis of the presence or not of P-bodies was made with a minimum of 100 cells, using Dcp2-GFP or Dhh1-GFP fluoresce as P-body markers.

\section{Accession numbers}

Gene expression omnibus (GEO) accession numbers for the whole experiment is GSE13100. All raw and processed data are held in the Valencia Yeast database (http://vydbase.uv.es).

\section{SUPPLEMENTAL MATERIAL}

Supplemental material can be found at http://www.rnajournal.org.

\section{ACKNOWLEDGMENTS}

We thank E. Herrero (tetO ${ }_{2}$ system), F. Navarro ( $r p b 1-1$ strain), and R. Parker (Dcp2-GFP and Dhh1-GFP plasmids) for their kind gift of strains and plasmids. We also thank F. Carrasco for technical help, J. Marín-Navarro for helping with the calculation of mRNA stabilities, and the rest of the members of the laboratory and Servicio de Chips de DNA-SCIE. We thank E. Herrero for critical reading of the manuscript. This work was supported by grants from the Spanish Ministerio de Educación y Ciencia BFU2005-08359 (to P.A.), BFU2007-67575-C03-01/BMC (to J.E.P.-O.), and BFU2006-07783 (to J.M.) and by a grant from the Generalitat Valenciana GV05/051 (to P.A.). P.A. was supported by the Ramón y Cajal Program. L.R.-S. was a recipient of a an F.P.I. fellowship from the Spanish Ministerio de Educación y Ciencia.

Received October 24, 2008; accepted February 27, 2009.

\section{REFERENCES}

Alepuz, P.M., Jovanovic, A., Reiser, V., and Ammerer, G. 2001. Stress induced MAP kinase Hog1 is part of transcription activation complexes. Mol. Cell 7: 767-777.

Alepuz, P.M., de Nadal, E., Zapater, M., Ammerer, G., and Posas, F. 2003. Osmostress-induced transcription by Hotl depends on a
Hog1-mediated recruitment of the RNA Pol II. EMBO J. 22: 2433 2442.

Bellí, G., Garí, E., Piedrafita, L., Aldea, M., and Herrero, E. 1998. An activator/repressor dual system allows tight tetracycline-regulated gene expression in budding yeast. Nucleic Acids Res. 26: 942-947.

Bhattacharyya, S., Habermacher, R., Martine, U., Closs, E., and Filipowicz, W. 2006. Relief of microRNA-mediated translational repression in human cells subjected to stress. Cell 125: 1111-1124.

Bond, U. 2006. Stressed out! Effects of environmental stress on mRNA metabolism. FEMS Yeast Res 6: 160-170.

Brengues, M., Teixeira, D., and Parker, R. 2005. Movement of eukaryotic mRNAs between polysomes and cytoplasmic processing bodies. Science 310: 486-489.

de Nadal, E., Alepuz, P.M., and Posas, F. 2002. Dealing with osmostress through MAP kinase activation. EMBO Rep. 3: 735-740.

Duttagupta, R., Tian, B., Wilusz, C.J., Khounh, D.T., Soteropoulos, P., Ouyang, M., Dougherty, J.P., and Peltz, S.W. 2005. Global analysis of Publp targets reveals a coordinate control of gene expression through modulation of binding and stability. Mol. Cell. Biol. 25: 5499-5513.

Escote, X., Zapater, M., Clotet, J., and Posas, F. 2004. Hog1 mediates cell-cycle arrest in G1 phase by the dual targeting of Sicl. Nat. Cell Biol. 6: 997-1002.

Fan, J., Yang, X., Wang, W., Wood, W.H., Becker, K.G., and Gorospe, M. 2002. Global analysis of stress-regulated mRNA turnover by using cDNA arrays. Proc. Natl. Acad. Sci. 99: 1061110616.

García-Martínez, J., Aranda, A., and Pérez-Ortín, J.E. 2004. Genomic run-on evaluates transcription rates for all yeast genes and identifies new gene regulatory mechanisms. Mol. Cell 15: 303313.

Garneau, N.L., Wilusz, J., and Wilusz, C.J. 2007. The highways and byways of mRNA decay. Nat. Rev. Mol. Cell Biol. 8: 113-126.

Gasch, A.P., Spellman, P.T., Kao, C.M., Carmel-Harel, O., Eisen, M.B., Storz, G., Botstein, D., and Brown, P.O. 2000. Genomic expression programs in the response of yeast cells to environmental changes. Mol. Biol. Cell 11: 4241-4257.

Greatrix, B.W. and van Vuuren, H.J. 2006. Expression of the HXT13, HXT15, and HXT17 genes in Saccharomyces cerevisiae and stabilization of the HXT1 gene transcript by sugar-induced osmotic stress. Curr. Genet. 49: 205-217.

Gowrishankar, G., Winzen, R., Dittrich-Breiholz, O., Redich, N., Kracht, M., and Holtmann, H. 2006. Inhibition of mRNA deadenylation and degradation by different types of cell stress. Biol. Chem. 387: 323-327.

Grigull, J., Mnaimneh, S., Pootoolal, J., Robinson, M.D., and Hughes, T.R. 2004. Genome-wide analysis of mRNA stability using transcription inhibitors and microarrays reveals post-transcriptional control of ribosome biogenesis factors. Mol. Cell. Biol. 24: 5534 5547.

Hilgers, V., Teixeira, D., and Parker, R. 2006. Translation-independent inhibition of mRNA deadenylation during stress in Saccharomyces cerevisiae. RNA 12: 1835-1845.

Hohmann, S., Krantz, M., and Nordlander, B. 2007. Yeast osmoregulation. Methods Enzymol. 428: 29-45.

Kawai, T., Fan, J., Mazan-Mamczarz, K., and Gorospe, M. 2004. Global mRNA stabilization preferentially linked to translational repression during the endoplasmic reticulum stress response. Mol. Cell. Biol. 24: 6773-6787.

Kedersha, N. and Anderson, P. 2007. Mammalian stress granules and processing bodies. Methods Enzymol. 431: 61-81.

Keene, J.D. and Tenenbaum, S.A. 2002. Eukaryotic mRNPs may represent posttranscriptional operons. Mol. Cell 9: 1161-1167.

Mata, J., Marguerat, S., and Bähler, J. 2005. Post-transcriptional control of gene expression: A genome-wide perspective. Trends Biochem. Sci. 30: 506-514.

Melamed, D., Pnueli, L., and Arava, Y. 2008. Yeast translational response to high salinity: Global analysis reveals regulation at multiple levels. RNA 14: 1337-1351. 


\section{Romero-Santacreu et al.}

Molina-Navarro, M.M., Castells-Roca, L., Bellí, G., GarcíaMartínez, J., Marín-Navarro, J., Moreno, J., Pérez-Ortín, J.E., and Herrero, E. 2008. Comprenhensive transcriptional analisis of the oxidative response in yeast. J. Biol. Chem. 283: 17908-17918.

Mumberg, D., Müller, R., and Funk, M. 1995. Yeast vectors for the controlled expression of heterologous proteins in different genetic backgrounds. Gene 156: 119-122.

O'Rourke, S.M. and Herskowitz, I. 2002. A third osmosensing branch in Saccharomyces cerevisiae requires the Msb2 protein and functions in parallel with the Shol branch. Mol. Cell. Biol. 22: 47394749.

Parker, R. and Sheth, U. 2007. P bodies and the control of mRNA translation and degradation. Mol. Cell 25: 635-646.

Pascual-Ahuir, A., Struhl, K., and Prof, M. 2006. Genome-wide location analysis of the stress-activated MAP kinase Hog1 in yeast. Methods 40: 272-278.

Pérez-Ortín, J.E., Alepuz, P.M., and Moreno, J. 2007. Genomics and gene transcription kinetics in yeast. Trends Genet. 23: 250-257.

Pokholok, D.K., Zeitlinger, J., Hannett, N.M., Reynolds, D.B., and Young, R.A. 2006. Activated signal transduction kinases frequently occupy target genes. Science 313: 533-536.

Posas, F., Chanbers, J.R., Heyman, J.A., Hoeffler, J., de Nadal, E., and Ariño, J. 2000. The transcriptional response of yeast to saline stress. J. Biol. Chem. 275: 17249-17255.

Proft, M. and Struhl, K. 2004. MAP kinase-mediated stress relief that precedes and regulates the timing of transcriptional induction. Cell 118: 351-361.

Proft, M., Mas, G., de Nadal, E., Vendrell, A., Noriega, N., Struhl, K., and Posas, F. 2006. The stress-activated Hog1 kinase is a selective transcriptional elongation factor for genes responding to osmotic stress. Mol. Cell 23: 241-250.

Rep, M., Krantz, M., Thevelein, J.M., and Hohmann, S. 2000. The transcriptional response of Saccharomyces cerevisiae to osmotic shock. J. Biol. Chem. 275: 8290-8300.

Rodríguez-Gabriel, M.A., Burns, G., McDonald, W.H., Martín, V., Yates III, J.R., Valer, J., and Russell, P. 2003. RNA-binding protein Csx1 mediates global control of gene expression in response to oxidative stress. EMBO J. 22: 6256-6266.

Sandler, H. and Stoecklin, G. 2008. Control of mRNA decay by phosphorylation of tristetraprolin. Biochem. Soc. Trans. 36: 491496.

Shalgi, R., Lapidot, M., Shamir, R., and Pilpel, Y. 2005. A catalog of stability-associated sequence elements in 3' UTRs of yeast mRNAs. Genome Biol. 6: R86. doi: 10.1186/gb-2005-6-10-r86.

Sheth, U. and Parker, R. 2003. Decapping and decay of messenger RNA occur in cytoplasmic processing bodies. Science 300: 805808.

Teixeira, D., Sheth, U., Valencia-Sánchez, M.A., Brengues, M., and Parker, R. 2005. Processing bodies require RNA for assembly and contain nontranslating mRNAs. RNA 11: 371-382.

Uesono, Y. and Toh-E, A. 2002. Transient inhibition of translation initiation by osmotic stress. J. Biol. Chem. 277: 1384813855.

Vasudevan, S. and Peltz, S.W. 2001. Regulated ARE-mediated mRNA decay in Saccharomyces cerevisiae. Mol. Cell 7: 1191-1200.

Wang, Y., Liu, C.L., Storey, J.D., Tibshirani, R.J., Herschlag, D., and Bown, P. 2002. Precision and functional specificity in mRNA decay. Proc. Natl. Acad. Sci. 99: 5860-5865. 

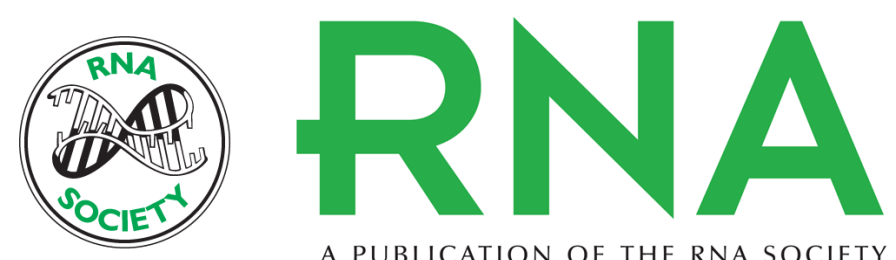

A PUBLICATION OF THE RNA SOCIETY

\section{Specific and global regulation of mRNA stability during osmotic stress in Saccharomyces cerevisiae}

Lorena Romero-Santacreu, Joaquín Moreno, José E. Pérez-Ortín, et al.

RNA 2009 15: 1110-1120 originally published online April 15, 2009

Access the most recent version at doi:10.1261/rna.1435709

Supplemental
Material http://rnajournal.cshlp.org/content/suppl/2009/04/16/rna.1435709.DC1

References This article cites 42 articles, 20 of which can be accessed free at: http://rnajournal.cshlp.org/content/15/6/1110.full.html\#ref-list-1

License

Email Alerting Receive free email alerts when new articles cite this article - sign up in the box at the Service top right corner of the article or click here. 